Z Rheumatol $2011 \cdot 70: 423-430$

DOI 10.1007/s00393-011-0824-4

(C) Springer-Verlag 2011

\section{Redaktion}

J. Wollenhaupt, Hamburg (Leitung)

O. Distler, Zürich

J. Grifka, Bad Abbach

G. Schett, Erlangen
H.R. Ziswiler · G. Caliezi · P.M. Villiger

Universitätsklinik für Rheumatologie, Klinische Immunologie und Allergologie, Inselspital, Bern

\title{
Muskuloskelettale Punktion, Injektion und Infiltration
}

\author{
Standpunkt von Schweizer Rheumatologen
}

\begin{abstract}
Zusammenfassung
Punktion, Injektion und Infiltration von Gelenken und Weichteilen gehören zu den grundlegenden Tätigkeiten der Rheumatologie. Da sich Indikationen und praktisches Vorgehen historisch entwickelt haben, ist die kritische Prüfung und ggf. die Anpassung etablierter Praktiken im Licht neuer wissenschaftlicher Erkenntnisse und technischer Errungenschaften zentral. Die wichtigsten Indikationen für eine Punktion sind die Infektsuche und die Druckentlastung bei Erguss. Gute Indikationen für Glukokortikoidinjektionen sind das entzündete, nichtinfizierte Gelenk und die aktivierte Arthrose. Kritisch hinterfragt werden müssen die seit Jahrzehnten etablierten, bis vor Kurzem kaum hinterfragten, im Einzelfall nicht selten repetitiv durchgeführten Infiltrationen bei mechanischer Enthesopathie am Epicondylus humeri radialis (Tennisellenbogen) oder der Plantarfaszie (Fasziitis plantaris). Bei diesen führen insbesondere wiederholte Glukokortikoidinfiltrationen zu einem schlechteren Verlauf als ohne Injektion.
\end{abstract}

\section{Schlüsselwörter}

Gelenkpunktion · Intraartikuläre Injektion · Glukokortikosteroide · Muskuloskelettales System • Tendinopathie

\section{Musculoskeletal puncture, injection and infiltration • Swiss rheumatologists' point of view}

- kostenfrei im Rahmen des jeweiligen

Zeitschriftenabonnements

- individuelle Teilnahme durch den Erwerb von CME.Tickets auf CME.springer.de

\section{Zertifizierung}

Diese Fortbildungseinheit ist mit 3 CME-Punkten zertifiziert von der Landesärztekammer Hessen und der Nordrheinischen Akademie für Ärztliche Fort- und Weiterbildung und damit auch für andere Ärztekammern anerkennungsfähig.

\section{Hinweis für Leser aus Österreich} Gemäß dem Diplom-Fortbildungs-Programm (DFP) der Österreichischen Ärztekammer werden die auf CME.springer.de erworbenen CME-Punkte hierfür 1:1 als fachspezifische Fortbildung anerkannt.

\section{Kontakt und weitere Informationen}

Springer-Verlag GmbH

Fachzeitschriften Medizin/Psychologie

CME-Helpdesk, Tiergartenstraße 17

69121 Heidelberg

E-Mail: cme@springer.com

CME.springer.de

\begin{abstract}
Arthrocentesis, injection and infiltration of joints and soft tissues belong to the basic procedures in rheumatology. The indications and the practical performance are based on experience and tradition. Nowadays, a crucial reappraisal and adaption of indications and technical aspects appear important in the light of new evidence and technical developments. The main indications for puncture remain the search of an infectious arthritis and reduction of intra-articular pressure due to effusion. Good indications for the injection of glucocorticoids are inflammation in sterile joints and activated osteoarthritis. The local infiltration with corticosteroids in mechanically induced enthesopathies at the lateral epicondyle of the humerus or at the plantar fascia have to be questioned in the light of recent publications which show that this common practice is associated with a poorer outcome than without injection.
\end{abstract}

\section{Keywords}

Joint puncture · Injections, intra-articular · Glucocorticoids · Musculoskeletal system · Tendinopathy 
Der Behandlungserfolg hängt von der korrekten Indikation, der korrekten Durchführung und dem Einsatz der geeigneten Medikamente ab
Die mit Abstand wichtigste Indikation für eine Punktion mit Aspiration ist die Infektsuche in der Synovialflüssigkeit

\section{Punctio sicca}

Trübes oder eitriges Punktat soll immer im Direktpräparat mitttels Gram-Färbung und Bakterienkulturen untersucht werden

\section{- Hämorrhagischer Erguss}

\section{Punktion, Infiltration und Injektion von Gelenken und Weichteilen sind Teil der täglichen Praxis jedes Rheumatologen. In den letzten Jahren warfen neue wissenschaftliche Er- kenntnisse zum Teil neues Licht auf etablierte Indikationen. Nach Lektüre dieses Artikels soll der Leser gute und schlechte Indikationen für Punktion, Infiltration und Injektion kennen, die häufigsten Risiken und Nebenwirkungen benennen können sowie über die theoretischen Grundlagen für das praktische Vorgehen informiert sein.}

Punktion, Injektion und Infiltration von Gelenken und Weichteilstrukturen (Bursae, Sehnenscheiden um Sehnen und Nerven) gehören seit mehr als 50 Jahren ins Arsenal der Methoden muskuloskelettaler Medizin. Wie bei allen anderen Prozeduren hängt deren Erfolg von der korrekten Indikation (bei wem/wann?), der korrekten Durchführung (wie?) und dem Einsatz der geeigneten Medikamente $\mathrm{ab}$ (womit?).

\section{Indikation}

\section{Punktion}

Die Gelenk (Bursa-/Sehnenscheiden)-Punktion führt bei großer Flüssigkeitsansammlung zu rascher Druckentlastung und Schmerzlinderung. Bereits Hippokrates hat über die Existenz von Gelenkflüssigkeit berichtet. Früheste Aufzeichnungen über Gelenkpunktionen reichen bis ins 16. Jahrhundert zurück (Fabricius Hildanus 1560-1634, „Tractado breve de medicina“, Mexico 1592; „Codex Badianus“, Aztekisches Manuskript). Die mit Abstand wichtigste Indikation für eine Punktion mit Aspiration ist die Infektsuche in der Synovialflüssigkeit. Sie muss bei jedem klinischen Verdacht auf Gelenk- oder Weichteilinfekt innerhalb von Stunden und vor Beginn einer antibiotischen Therapie erzwungen werden. Bei Unsicherheit über die vorhandene Flüssigkeitsmenge und deren Lokalisation oder nach misslungenem Punktionsversuch gilt heute die ultraschallgesteuerte Punktion als Methode der Wahl [15]. Bei - Punctio sicca ist bei korrekter Kanülenplatzierung eine Lavage indiziert. Trübes oder gar eitriges Punktat soll immer im Direktpräparat mit einer Gram-Färbung und mittels Bakterienkulturen untersucht werden. Ebenfalls im Direktpräparat können unter dem Polarisationsmikroskop Harnsäure- und/oder Kalziumpyrophospatkristalle identifiziert werden (• Abb. 1). Deren Nachweis schließt einen Infekt nicht aus. Andererseits ist das routinemäßige Anlegen von Kulturen auf Bakterien aus Gelenkflüssigkeit bei fehlendem Infektverdacht und klarer Flüssigkeit weder kosteneffizient noch indiziert. Die Zellzählung und allenfalls deren Differenzierung in der Synovialflüssigkeit geben starke Hinweise darauf, ob ein rein mechanisches Problem im Sinne der Degeneration (Zellzahl 0 bis max. einige Hundert), ein leicht entzündlicher Prozess (Zellzahl 1000 bis ca. 10.000) oder ein hoch inflammatorisches Geschen abläuft (mehrere 10.000 bis 100.000 Zellen).

Kleinste (oft iatrogene) Blutbeimengungen zum Punktat führen bereits zu einer Rotverfärbung, die den Untersucher nicht beunruhigen sollte - dies im Gegensatz zum $>$ hämorrhagischen Erguss, insbesondere wenn darin sogar noch Fettaugen zu erkennen sind. Im diesem Fall muss an einen Hämarthros bei hämorrhagische Diathese oder an eine intraartikuläre Fraktur (Fett durch Eröffnung des Markraums) gedacht werden.
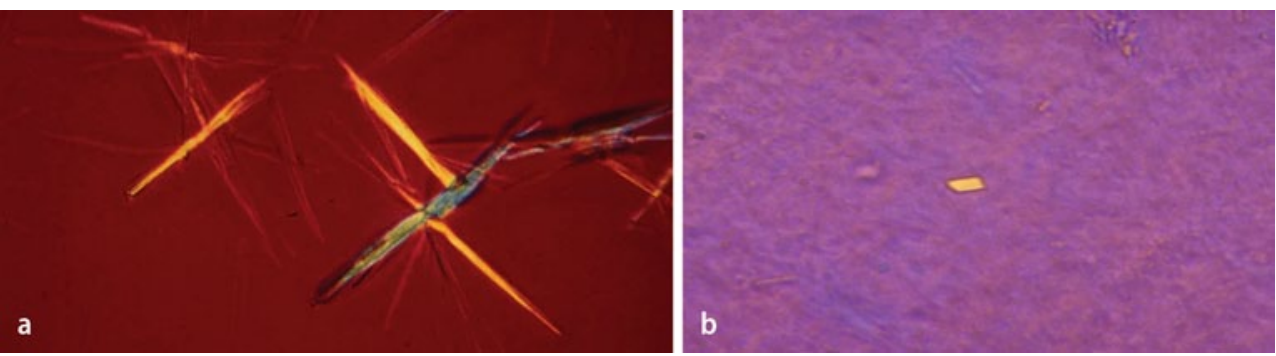

Abb. 1 A Kristallnachweis aus der Synovia mit dem Polarisationsmikroskop: a Urat (Gicht)-Nadeln, b Kalziumpyrophosphat 


\begin{tabular}{l} 
Tab. 1 Gute Indikationen für Punktion/ \\
Injektion \\
\hline Punktion \\
\hline - Suche/Ausschluss Infekt (Notfallindikation!) \\
\hline - Gelenkentlastung \\
\hline Punktion und Injektion/Infiltration \\
\hline $\begin{array}{l}\text { Entzündliche Arthritiden und Tenosynovitiden/ } \\
\text { Bursitiden }\end{array}$ \\
\hline - rheumatoide Arthritis, juvenile Arthritiden \\
\hline - kristallinduzierte Arthritis (Gicht/Pseudogicht) \\
\hline - Spondyloarthritiden (Spondylitis ankylosans, \\
Psoriasisarthritis, reaktive Arthritis, undiffe- \\
renzierte SpA) \\
\hline $\begin{array}{l}\text { Nichtentzündliche Gelenk- und Weichteilerkran- } \\
\text { kungen }\end{array}$ \\
\hline - reaktivierte Arthrose: Knie, Fingergelenke, \\
Hüfte, Akromioklavikulargelenk \\
\hline - subakromiale Einklemmungsproblematik (Bur- \\
sitis subacromialis, Tendinose, Kalkschulter) \\
\hline - adhäsive Kapsulitis der Schulter (,,frozen \\
shoulder") \\
\hline - Tenosynovitis stenosans (nodularis): sprin- \\
gender Finger \\
\hline - Karpaltunnelsyndrom, Morton-Metatarsalgie \\
\hline - Tendovaginitis de Quervain \\
\hline - Bursitis trochanterica \\
\hline
\end{tabular}

schleunigten Krankheitsprogression $[1,12]$. Bei Hüftarthrose dient die im Ultraschall dargestellte Synovialitis als Biomarker für das Ansprechen auf eine Glukokortikoidinjektion. Nach ultraschallgesteuerter Injektion einer Hüftarthrose kann mit einem sehr raschen Ansprechen und mit 8 Wochen Wirkdauer gerechnet werden; dies ist der Injektion mit Kochsalzlösung und jener mit einem Hyaluronsäurepräparat signifikant überlegen [2]. Intraartikuläre Kortikosteroidinjektionen bei Rhizarthrose [10] und Omarthrose sind gemäß publizierter Daten weniger effizient [3].

Bei \ subakromialer Einklemmungsproblematik sollte dem Behandlungskonzept immer eine möglichst genaue funktionelle und morphologische Beurteilung zugrunde gelegt werden. Die Behandlung umfasst praktisch immer eine aktive physikalische Therapie zur besseren Stabilisation glenohumoral; v. a. initial scheitert diese jedoch oft wegen zu starker Schmerzen. Systematische Reviews zur Behandlung subakromialer Schmerzen, der subakromialen Einklemmung und von Rotatorenmanschettensyndromen favorisieren subakromiale Injektionen gegenüber ergonomischen Anpassungen, kortisonfreien Entzündungshemmern, Akupunktur, Beweglichkeits- und Stabilisationsübungen, Eis- und Wärmeapplikation sowie Physiotherapie [3]. Die subakromiale Injektion ist hilfreich für die Differenzierung zwischen Schulterschwäche, verursacht durch eine reine Einklemmungsproblematik (Kraft verbessert sich nach der Injektion), und einer Rotatorenmanschettenruptur (keine Verbesserung der Kraft nach der Injektion).

Bei $\$ adhäsiver Kapsulitis der Schulter sollte früh eine intraartikuläre Glukokortikoidinjektion ins Auge gefasst werden. Im Vergleich zu Physiotherapie und alleiniger Instruktion von Beweglichkeitsübungen führen intraartikuläre Glukokortikoide zu signifikant besserer Schmerzreduktion und Funktionsverbesserung nach 6 Wochen und 3 Monaten. Nach einem Jahr sind die Resultate unabhängig von der initial angebotenen Therapieform günstig [4].

Die De-Quervain-Tenosynovitis am 1. Strecksehnenfach kann in den meisten Fällen mit einer Glukokortikoidinfiltration kurativ behandelt werden. Sie ist der Behandlung mit kortisonfreien Entzündungshemmern, der Ruhigstellung mit Schiene oder deren Kombination überlegen [11].

Die Tenosynovitis stenosans an den Fingern II bis V beruht auf einer relativen Stenosierung in der Regel auf Höhe des A1-Ringbandes, meistens bedingt durch eine Verdickung dieses anulären Bandes. Auch sie spricht in der Mehrzahl der Fälle sehr gut auf eine lokale Glukokortikoidinfiltration an und sollte in jedem Fall vor chirurgischer Intervention in Betracht gezogen werden.
Eine residuelle mono- oder oligoartikuläre Aktivität einer stabilen primär entzündlichen Gelenkerkrankung ist eine gute Indikation für eine intraartikuläre Glukokortikoidbehandlung

\section{$\rightarrow$ Reaktivierte Arthrose}

Bei Hüftarthrose dient die im Ultraschall dargestellte Synovialitis als Biomarker für das Ansprechen auf eine Glukokortikoidinjektion

Subakromiale Einklemmungs-
problematik

- Adhäsive Kapsulitis der Schulter

De-Quervain-Tenosynovitis

- Tenosynovitis stenosans 

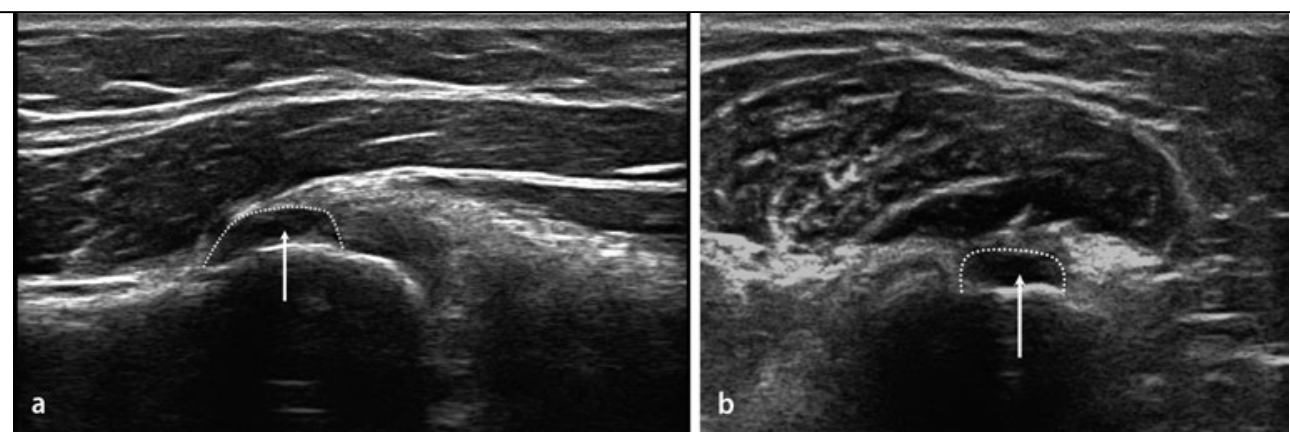

Abb. $2 \Delta$ Ultraschall an der Enthese am Epicondylus humeri lateralis bei „Tennisellenbogen“: Darstellung einer Partialruptur (a Längs-, b Transversalschnitt)
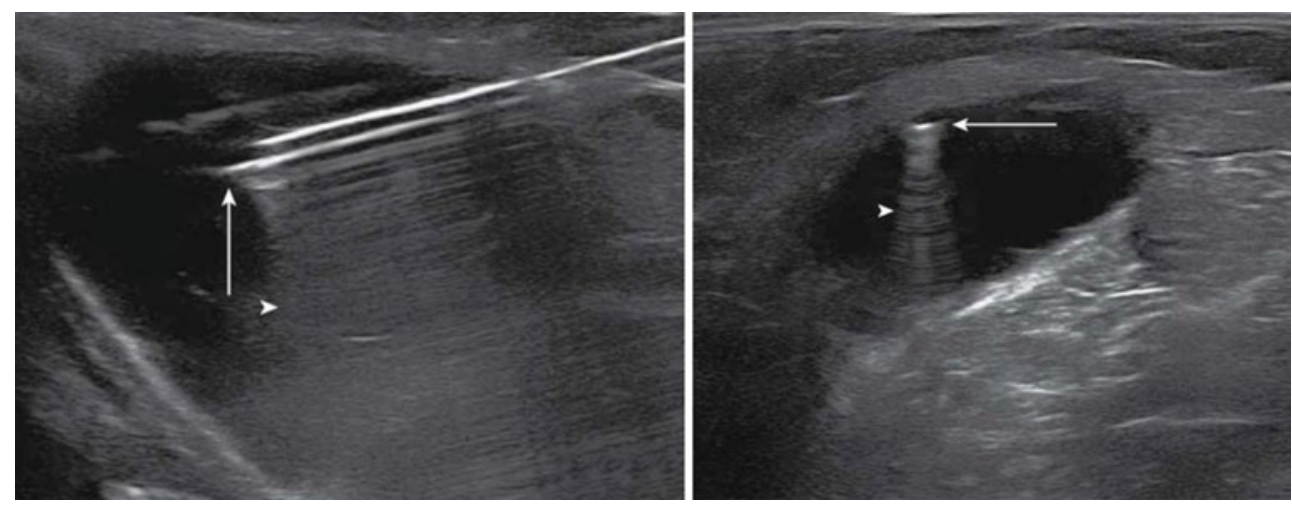

Abb. $3 \Delta$ Kontrolle der korrekten Nadellage mittels Ultraschall in einer Baker-Zyste (Pfeil Nadel, Pfeilspitze Wiederholungsartefakt; a Längs-, b Transversalschnitt)

\section{Karpaltunnelsyndrom}

\section{Morton-Metatarsalgie}

\section{Enthesopathie der Plantarfaszie}

Je häufiger Glukokortikoide infiltriert werden, desto schlechter wird das Resultat
Das $\$ Karpaltunnelsyndrom ist die häufigste Einklemmungsneuropathie mit einer Lebenszeitprävalenz von 4\%. Die lokale Glukokortikoidinfiltration lindert die Symptome signifikant besser als eine Placeboinfiltration [„number needed to treat" $(\mathrm{NNT})=2$ ] und orale Glukokortikoide. Lokale Glukokortikoide sind verglichen mit der chirurgischen Dekompression nach 3 Monaten signifikant häufiger effektiv, nach 6 Monaten ebenbürtig und nach 12 Monaten im Vergleich zur initialen Chirurgie etwas weniger häufig wirksam (Unterschiede nach 12 Monaten nicht signifikant; [9]).

Wie beim Karpaltunnelsyndrom lindert auch bei der $\$ Morton-Metatarsalgie häufig bereits eine einmalgie Glukokortikoidinfiltration ausreichend [18]. Bei beiden Einklemmungsneuropathien kann als sonographisch äußerst zuverlässiges Zeichen eine olivenförmige Verdickung des Nervs als Reaktion auf die Druckeinwirkung dargestellt werden $[18,20]$.

\section{Umstrittene Indikationen für therapeutische Infiltrationen}

Tendinopathien und Enthesopathien, verursacht durch mechanische Überbelastung („overuse“) insbesondere am Epicondylus humeri lateralis (Tennisellenbogen)/medialis (Golferellenbogen) und die Enthesopathie der Plantarfaszie (Fasziitis plantaris) wurden bis vor Kurzem als klare Indikationen für lokale Glukokortikoidinfiltrationen angesehen. Histologisch sind diese Läsionen gekennzeichnet durch angiofibroplastische Hyperplasie, Hyperzellularität, Neovaskularisation, erhöhte Proteinsynthese und gestörte Matrixorganisation, aber Fehlen von Zeichen aktiver Entzündung [8]. Mittels Weichteilbildgebung (MRT und Ultraschall) sind häufig Störungen der Fibrillentextur bis hin zu Sehnenfaserrissen dokumentierbar ([16], • Abb. 2). Allein diese Beobachtungen verlangen eine kritische Prüfung der Wirksamkeit lokaler Glukokortikoidinfiltrationen.

Die lokale Glukokortikoidinfiltration bei lateraler Epikondylopathie ist zwar kurzfristig wirksamer als keine Therapie, Physiotherapie und Placeboinfiltration, führt jedoch mittelfristig (3-6 Monate) und langfristig (12 Monate) zu eindeutig schlechteren Resultaten als die anderen genannten Modalitäten. Je häufiger Glukokortikoide infiltriert werden, desto schlechter wird das Resultat [6, 14]. In Analogie dürfte die Argumentation betreffend medialer Epikondylopathie, Enthesopathie der Plantarfaszie und Tendinopathie des Achillessehnenkörpers lauten, auch wenn wissenschaftliche Evidenz dafür (noch) fehlt.

Kontraindikationen für lokale Injektionen sind in $\bullet$ Tab. 2 aufgelistet. 
Tab. 2 Kontraindikationen für lokale

Injektionen

Hautverletzungen oder Infektion an der

Punktions-/Injektionsstelle

Fraktur des Gelenks

Instabile Antikoagulation

Unverträglichkeit/Allergie gegenüber zu

applizierenden Medikamenten

Gelenkprothesen $^{\mathrm{a}}$

a Relative Kontraindikation (hohe Anforderun-

gen für Asepsis, in Deutschland Injektion unter

Operationsbedingungen).

Tab. 3 Komplikationen nach Gelenk-

und Weichteilinfiltrationen

"Flare"

Vasovagale Reaktion

Gesichtsrötung , flush"

Gelenkinfektion

Hypersensitivitätsreaktion

Zyklusunregelmäßigkeiten

Blutzuckerschwankungen (Diabetiker)

Weichteilverkalkung

Sehnenruptur

Hautatrophie und -depigmentierung
Tab. 4 Praktisches Vorgehen

Indikationsstellung

Einwilligung des Patienten mündlich

(Schweiz) oder schriftlich

Geschlossener Raum ohne Luftzug

Tragen von Mundschutz aller Personen im

\section{Raum}

Tragen von nichtsterilen Handschuhen (in

Deutschland Verwendung steriler Hand-

schuhe)

Bequeme Positionierung von Patient und Arzt

Lokalisation und Markierung der Injektions-

stelle

Vermeiden von oberflächlichen Venen

Desinfektion mit Alkohol, mindestens 1 min

einwirken lassen

"No-touch"-Technik

Aspiration der Flüssigkeit

Injektion der Medikamente (Cave: Widerstand)

Kompression der Injektionsstelle

Wundschnellverband

\section{Patienteninformation, Risiken, Komplikationen}

Der Patient muss über die Indikation zur Intervention, über Risiken und Nebenwirkungen (• Tab. 3) sowie Behandlungsalternativen aufgeklärt werden. Nach lokaler Glukokortikoidinjektion/-infiltration sind ein Aufflammen der Beschwerden („post-injection flare“) sowie eine Überwärmung und Rötung des Gesichts („flush“; [7]) am häufigsten beschrieben. Selten tritt eine lokale Haut- oder Fettatrophie auf (1\%), wobei solche am ehesten nach (zu) oberflächlichen Injektionen im Hand-/Fingerbereich gesehen werden. Bei Infiltrationen um die Achillessehne oder Plantarfaszie muss an das Risiko einer Sehnenruptur gedacht werden $(<1 \%)$.

Unmittelbar um die Injektion/Infiltration müssen Arzt und Patient auf vasovagale Reaktionen und Hypersensitivitätsreaktionen gefasst sein. Die stabile Lagerung des Patienten auf einer Liege kann daher von Vorteil sein, ist jedoch bei Injektionen an Fingergelenken oder der Schulter nicht immer sinnvoll und unpraktisch. Prämenopausale Frauen erleben nach Glukokortikoidinfiltrationen gelegentlich Störungen im Menstruationszyklus. Iatrogene Infektionen nach Gelenkinjektionen und Weichteilinfiltrationen sind sehr selten (<1:100.000 bis 1:78.000; $[5,13,17])$. Gleichwohl ist der Patient über diese Komplikation zu informieren, ebenso über die seltenen lokalen Blutungen oder Nervenverletzungen (v. a. im Bereich der Finger beschrieben). Werden mit den Glukokortikoiden gleichzeitig auch Lokalanästhetika verabreicht, lohnt es sich, dem Patienten mitzuteilen, dass die Beschwerden mit dem Abklingen der Lokalanästhesie wieder stärker auftreten können, diese sich aber durch die eintretende Wirkung der Steroide nach 24 bis 48 Stunden wieder bessern. Bei Diabetikern ist nach Weichteilinfiltrationen mit Glukokortikoiden - weniger häufig nach Gelenkinjektionen - mit Blutzuckerschwankungen über mehrere Tage bis zu 3 Wochen zu rechnen. Diese Effekte sind interindividuell sehr variabel und können im Einzelfall nicht vorhergesagt werden. Engmaschigere Blutzuckerkontrollen sind daher angezeigt [19].

\section{Praktisches Vorgehen für die Punktion/Injektion/Infiltration}

Die Information über Indikation und Risiken der Intervention sowie das Einverständnis des Patienten sollen schriftlich in der Krankenakte festgehalten werden. Es bewährt sich, das routinemä-

latrogene Infektionen nach Gelenkinjektionen und Weichteilinfiltrationen sind sehr selten

Die Information über Indikation und Risiken der Intervention sowie das Einverständnis des Patienten sollen schriftlich in der Krankenakte festgehalten werden 


\section{"No-touch“-Technik}

Die Wahl der Nadel hängt von der zu punktierenden Struktur und der Absicht ab

Vor der Injektion von Glukokortikoiden wird der Widerstand gepürft

\begin{tabular}{|c|c|c|c|}
\hline Gelenk & $\begin{array}{l}\text { Steroiddosis } \\
\text { Triamcinolonacetat }(\mathrm{mg})\end{array}$ & $\begin{array}{l}\text { Injektionsvolumen } \\
\text { (ml) }\end{array}$ & $\begin{array}{l}\text { Nadeldicke } \\
\text { (Gauge) }\end{array}$ \\
\hline Schulter & 40 & $5-10$ & $21(20)$ \\
\hline Ellenbogen & 20 & $3-5$ & $23(21)$ \\
\hline Handgelenk & 20 & 3 & $23(21)$ \\
\hline Finger/Zehen & 5 & 0.25 & 30 \\
\hline Knie & 40 & $5-10$ & $18(-21)$ \\
\hline Sprunggelenk & 20 & $2-3$ & $21(23)$ \\
\hline \multicolumn{4}{|c|}{$\begin{array}{l}\text { Je kleiner das Injektionsvolumen, desto höher die Glukokortikoidkonzentration (Beispiel: Das Verwenden von } 40 \text { mg Kenakort- } \\
\text { ampullen für Fingergelenke ergibt ein gewünscht kleines Injektionsvolumen). }\end{array}$} \\
\hline
\end{tabular}

Bige Vorgehen (• Tab. 4) in einer Klinik- oder Praxisrichtlinie schriftlich zu dokumentieren; entsprechende Richtlinien der Fachgesellschaften für Rheumatologie der Schweiz und von Deutschland sind online verfügbar (http://www.rheuma-net.ch/Richtlinien; http://dgrh.de/qualitaetsmanual2.html). Diese Richtlinien decken sich nicht im Punkt zum Einsatz steriler bzw. unsteriler Handschuhe, weshalb wir den Lesern empfehlen, die Gepflogenheiten in ihrem Land zu berücksichtigen.

Im Interventionsraum sollen nur so viele Personen wie nötig anwesend sein (in der Regel Arzt und Patient); diese tragen eine Gesichtsmaske. Fenster und Türen werden geschlossen. Sterile Handschuhe bringen bei konsequenter "No-touch“-Technik keine Vorteile. (Nichtsterile) Handschuhe dienen in erster Linie dem Arzt als Schutz vor allfälligen Infekten des Patienten (z. B. HIV, Hepatitis).

Vor der Hautdesinfektion wird die Punktionsstelle mittels Druckmarke (z. B. Kugelschreiber) oder mit Stift (Fadenkreuz) markiert. Alkoholische Lösungen für die Hautdesinfektion sind jodhaltigen ebenbürtig und werden heute an den meisten Institutionen bevorzugt. Entscheidend für Effizienz der Desinfektion ist nicht eine bestimmte Wisch- oder Auftragetechnik, sondern in erster Linie die genügend lange Einwirkzeit $(\geq 60 \mathrm{~s})$. Die zu desinfizierende Hautregion muss ausreichend angefeuchtet werden; am einfachsten ist das Auflegen einer mit Desinfektionmittel gut genässten Longuette (dadurch ist der gleichzeitige Schutz vor Anflugkeimen gewährleistet). Das Abdecken mit sterilen Tüchern ist zu unterlassen. Es spiegelt eine falsche Sicherheit vor, ist zeitaufwendig und kostenintensiv.

Eine Lokalanästhesie der Einstichstelle ist bei Erwachsenen selten nötig, bei Injektionen in Fingergelenke verwenden wir steriles Kältespray mit Chlorethyl unmittelbar vor der Injektion. Bei geplanten Punktionen und Injektionen bei Kindern hat sich die Anwendung von lokalanästhesierender Creme oder Pflaster (EMLA) bewährt. Es muss dafür allerdings vor der Intervention eine Einwirkzeit von 60 bis 90 Minuten eingerechnet werden. Die Wahl der Nadel hängt von der zu punktierenden Struktur und der Absicht ab. Ist das Ziel die Aspiration von Gelenkflüssigkeit, wird eine möglichst dicke Nadel gewählt; ist nur eine Injektion/Infiltration vorgesehen, verwendet man möglichst dünne Nadeln. Nach erfolgter Desinfektion werden die Einstichstelle und deren desinfizierte Umgebung nicht mehr berührt (sog. „No-touch“-Technik). Bei Gelenkpunktionen wird die Synovia möglichst ohne großen Sog aspiriert und nach Entleerung des Gelenks die Nadel in situ belassen. Vor der Injektion von Glukokortikoiden wird der Widerstand geprüft. Die korrekte Lage wird erkennbar, indem noch Restflüssigkeit (oder Injektat) aspiriert werden kann und/oder der Widerstand für die Injektion niedrig ist. Im Zweifelsfall und bei Verfügbarkeit eines Geräts kann die Nadel mittels Ultraschall positioniert werden (• Abb. 3). Im Anschluss an die Intervention wird ein Wundschnellverband aufgeklebt; dieser dient v. a. dem Schutz der Kleidung und der Umgebung des Patienten, falls es noch leicht nachbluten sollte. Kleine Fingergelenke sollten unter Zug (Erzeugen eines Unterdrucks im Gelenk) injiziert werden. Ferner sollte die Punktionsstelle nach Injektion während etwa 60 Sekunden komprimiert werden (Verhindern eines Rückfließens von Glukokortikoiden mit Gefahr einer Hautatrophie).

\section{Medikamente}

Die intraartikuläre Applikation von Glukokortikoiden ( $\bullet$ Tab. 5) führt neben der Verminderung der lokalen Entzündungsreaktion auch zu einem reduzierten synovialen Blutfluss und einer veränderten Kollagensynthese. Wir empfehlen, bei fehlenden Kontraindikationen wie etwa allergischen Reaktionen immer ein $>$ Lokalanästhetikum beizumischen. Dies bringt nicht nur dem Patienten 
unmittelbare Erleichterung, sondern gibt auch wertvolle Informationen betreffend korrekter Technik (z. B. Gelenkanästhesie) und korrekter Diagnose (z. B. koxogene vs. vertebrogene Oberschenkelschmerzen).

Die Wirkstoffe der handelsüblichen Präparate sind Betamethasonnatriumphosphat, Triamcinolonhexacetonid und -acetat, Methylprednisolonacetat und Dexamethasonhydrogenphosphat. Während Betamethason und Dexamethason gut löslich sind und dementsprechend gut diffundieren, sind Triamcinolon und Methylprednisolon schlechter löslich und verbleiben länger am Ort der Infiltration, was im Falle eine intraartikulären Applikation gewünscht ist. Trimacinolonhexacetonid hat die längste Verweil- und Wirkdauer. Es gibt keine Evidenz für eine anhaltende Wirksamkeit löslicher Glukokortikoide. Wir empfehlen deshalb eine sehr restriktive diesbezügliche Haltung. In den meisten Fällen kann und soll mit $>$ kristallinen Präparaten behandelt werden. In der Regel sind Patienten nach einer technisch einwandfreien Injektion/Infiltration länger andauernd beschwerdefrei (Bursitis trochanterica, Tendovaginitis de Quervain). Die Frequenz der Interventionen richtet sich in erster Linie nach der Indikation. Die oft genannten 4 Wiederholungen pro Jahr entsprechen einer tradierten Meinung, die jeglicher evidenzbasierter Grundlage entbehrt. Bei einer hartnäckigen Arthritis können repetitive, engmaschige Injektionen indiziert sein; bei einer asymptomatischen Arthrose wäre bereits eine Injektion eine zuviel.

\section{Korrespondenzadresse \\ PD Dr. H.R. Ziswiler}

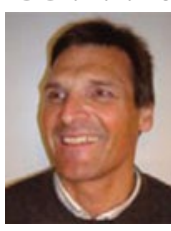

Universitätsklinik für Rheumatologie, Klinische Immunologie und Allergologie, Inselspital

$\mathrm{CH}-3010$ Bern

hansruedi.ziswiler@insel.ch

Interessenkonflikt. Der korrespondierende Autor gibt an, dass kein Interessenkonflikt besteht.

\section{Kristalline Präparate}

In der Regel sind Patienten nach einer technisch einwandfreien Injektion/ Infiltration länger andauernd beschwerdefrei

\section{Literatur}

1. Arroll B, Goodyear-Smith F (2004) Corticosteroid injections for osteoarthritis of the knee: meta-analysis. BMJ 328:869

2. Atchia I, Kane D, Reed MR et al (2011) Efficacy of a single ultrasound-guided injection for the treatment of hip osteoarthritis. Ann Rheum Dis 70:110-116

3. Buchbinder R, Green S, Youd JM (2003) Corticosteroid injections for shoulder pain. Cochrane Database Syst Rev:CD004016

4. Carette $\mathrm{S}$, Moffet $\mathrm{H}$, Tardif J et al (2003) Intraarticular corticosteroids, supervised physiotherapy, or a combination of the two in the treatment of adhesive capsulitis of the shoulder: a placebo-controlled trial. Arthritis Rheum 48:829-838

5. Charalambous $C P$, Tryfonidis M, Sadiq S et al (2003) Septic arthritis following intra-articular steroid injection of the knee - a survey of current practice regarding antiseptic technique used during intra-articular steroid injection of the knee. Clin Rheumatol 22:386-390
6. Coombes BK, Bisset L, Vicenzino B (2010) Efficacy and safety of corticosteroid injections and other injections for management of tendinopathy: a systematic review of randomised controlled trials. Lancet 376:17511767

7. Courtney P, Doherty M (2009) Joint aspiration and injection and synovial fluid analysis. Best Pract Res Clin Rheumatol 23:161-192

8. Khan KM, Cook JL, Kannus P et al (2002) Time to abandon the "tendinitis" myth. BMJ 324:626-627

9. Ly-Pen D, Andréu JL, De Blas G et al (2005) Surgical decompression versus local steroid injection in carpal tunnel syndrome: a one-year, prospective, randomized, open, controlled clinical trial. Arthritis Rheum 52:612-619

10. Meenagh GK, Patton J, Kynes C et al (2004) A randomised controlled trial of intra-articular corticosteroid injection of the carpometacarpal joint of the thumb in osteoarthritis. Ann Rheum Dis 63:1260-1263
11. Peters-Veluthamaningal C, Van Der Windt DA, Winters JC et al (2009) Corticosteroid injection for de Quervain's tenosynovitis. Cochrane Database Syst Rev:CD005616

12. Raynauld JP, Buckland-Wright $C$, Ward R et al (2003) Safety and efficacy of long-term intraarticular steroid injections in osteoarthritis of the knee: a randomized, double-blind, placebo-controlled trial. Arthritis Rheum 48:370-377

13. Seror $P$, Pluvinage $P$, $D$ 'andre $F L$ et al (1999) Frequency of sepsis after local corticosteroid injection (an inquiry on 1160000 injections in rheumatological private practice in France). Rheumatology (Oxford) 38:12721274

14. Smidt N, Van Der Windt DA, Assendelft WJ et al (2002) Corticosteroid injections, physiotherapy, or a waitand-see policy for lateral epicondylitis: a randomised controlled trial. Lancet 359:657-662

15. Tamborrini G, Backhaus M, Schmidt W et al (2010) Ultrasound and arthritis. Z Rheumatol 69:889-902
16. Walz DM, Newman JS, Konin GP et al (2010) Epicondylitis: pathogenesis, imaging, and treatment. Radiographics 30:167-184

17. Weston VC, Jones AC, Bradbury N et al (1999) Clinical features and outcome of septic arthritis in a single UK Health District 1982-1991. Ann Rheum Dis 58:214-219

18. Wu KK (2000) Morton neuroma and metatarsalgia. Curr Opin Rheumatol 12:131-142

19. Younes $M$, Neffati F, Touzi M et al (2007) Systemic effects of epidural and intra-articular glucocorticoid injections in diabetic and non-diabetic patients. Joint Bone Spine 74:472476

20. Ziswiler HR, Reichenbach S, Vögelin E et al (2005) Diagnostic value of sonography in patients with suspected carpal tunnel syndrome: a prospective study. Arthritis Rheum 52:304311 


\section{CME-Fragebogen}

\section{kostenfreie Teilnahme für Abonnenten}

Bitte beachten Sie:

- Antwortmöglichkeit nur online unter: CME.springer.de

- Die Frage-Antwort-Kombinationen werden online individuell zusammengestellt.

- Es ist immer nur eine Antwort möglich.

\section{Welches ist die wichtigste Indikation für eine Gelenk- punktion? \\ $\square$ Suche einer Kristall- arthropathie \\ $\square$ Entlastung vom Überdruck \\ $\square$ Ausschluss von/Suche nach septischer Arthritis \\ $\square$ Diagnose einer rheumatoiden Arthritis \\ $\square$ Diagnose einer Lyme-Borre- liose}

\section{Bei klarer Kniegelenkflüssig- keit mit einer Zellzahl von 200/ $\mu$ list ... \\ $\square$ eine Kultur auf Bakterien dringend nötig. \\ $\square$ am ehesten von einem mechanischen Reizerguss auszugehen. \\ $\square$ eine Kristallarthritis im Schub sehr wahrscheinlich. \\ $\square$ eine hoch infektiöse Arthritis vorhanden. \\ $\square$ eine Arthrose ausgeschlossen.}

Wiederholte lokale Kortikosteroidinfiltrationen sind nicht vertretbar bei ...

$\square$ einer aktivierten Arthrose des Knies.

$\square$ einem monoartikulären Schub bei rheumatoider Arthritis.

$\square$ einem Tennisellenbogen.

$\square$ einer akuten Gichtarthritis.

$\square$ einer adhäsiven Kapsulitis der Schulter.

Bei adhäsiver Kapsulitis der Schulter sind intraartikuläre Kortikosteroide ...

$\square$ kontraindiziert.

$\square$ erst spät im Verlauf sinnvoll.

$\square$ früh im Verlauf sinnvoll. nicht wirksam.

im Verlauf nach 12 Monaten allen anderen Therapieformen überlegen.

\section{Bei Tenosynovitis de Quer-} vain ...

$\square$ muss fast immer operiert werden.

$\square$ ist das 3. Strecksehnenfach betroffen.

$\square$ ist die lokale Kostikosteroidinjektion eine sehr wirksame Maßnahme.

$\square$ ist die Ruhigstellung auf der Schiene die wirksamste Behandlung.

$\square$ sind kortisonfreie Entzündungshemmer die wirksamste Behandlung.

Die lokale Kortikosteroidinfiltration beim Karpaltunnelsyndrom ...

$\square$ hilft weniger gut als orale Kortikosteroide.

$\square$ hilft nie.

$\square$ ist immer besser als die Operation.

$\square$ führt nach 3 Monaten zu signifikant besseren Resultaten als die Operation.

$\square$ führt nach 12 Monaten zu signifikant schlechteren Resultaten als die Operation.

\section{Die häufigste Nebenwirkung} nach lokaler Kortikosteroidinfiltration ist ...

$\square$ die iatrogene Infektion. $\square$ eine Blutzuckerentgleisung.

$\square$ eine Haut- und Fettatrophie.

$\square$ eine Sehnenruptur.

$\square$ ein Aufflammen der Beschwerden („flare").
Das Standardvorgehen für eine Punktion/Infiltration eines

Gelenks umfasst:

$\square$ Abrasieren der Haare

$\square$ Desinfektion mindestens $30 \mathrm{~s}$ lang

$\square$ Sterile Abdeckung

$\square$ "No-touch"-Technik

$\square$ Anschließender

Kompressionsverband

Das Kortikosteroidpräparat mit der längsten lokalen Verweil- und Wirkdauer ist ...

$\square$ Betamethasonnatriumphosphat.

$\square$ Triamcinolonacetat.

$\square$ Methylprednisolonacetat.

$\square$ Triamcinolonhexacetonid.

$\square$ Dexamethasonhydrogenphosphat.

\section{Die korrekte Nadellage ...}

$\square$ ist erkennbar an einem hohen Widerstand bei der Infiltration.

$\square$ kann im Zweifelsfall mittels Ultraschallkontrolle verifiziert werden.

$\square$ wird bestätigt, wenn Blut aspiriert werden kann.

$\square$ kann immer durch Aspiration von Gelenkflüssigkeit bestätigt werden.

$\square$ ist belanglos.

Diese Fortbildungseinheit ist

12 Monate auf

CME.springer.de verfügbar.

Den genauen Einsendeschluss

erfahren Sie unter

CME.springer.de 